\title{
Moderate calorie restriction during gestation programs offspring for lower BAT thermogenic capacity driven by thyroid and sympathetic signaling
}

\author{
M Palou ${ }^{1}$, T Priego ${ }^{1}$, M Romero ${ }^{2}$, N Szostaczuk ${ }^{1}$, J Konieczna $^{1}$, C Cabrer $^{1}$, X Remesar $^{2}$, A Palou $^{1}$ and C Pico ${ }^{1}$
}

BACKGROUND: Maternal calorie restriction during pregnancy programs offspring for later overweight and metabolic disturbances. Brown adipose tissue (BAT) is responsible for non-shivering thermogenesis and has recently emerged as a very likely target for human obesity therapy.

OBJECTIVE: Here we aimed to assess whether the detrimental effects of undernutrition during gestation could be related to impaired thermogenic capacity in BAT and to investigate the potential mechanisms involved.

METHODS: Offspring of control and $20 \%$ calorie-restricted rats (days 1-12 of pregnancy) (CR) were studied at the age of 25 days. Protein levels of uncoupling protein 1 (UCP1) and tyrosine hydroxylase (TyrOH); mRNA levels of lipoprotein lipase (LPL), carnitine palmitoyltransferase 1 (CPT1) and deiodinase iodothyronine type II (DIO2) in BAT; and blood parameters including thyroid hormones, were determined. The response to 24-h cold exposure was also studied by measuring body temperature changes over time, and final BAT UCP1 levels.

RESULTS: Compared with controls, CR animals displayed in BAT lower UCP1 and TyrOH protein levels and lower LPL and CPT1 mRNA levels; they also showed lower triiodothyronine (T3) plasma levels. CR males, but not females, revealed lower DIO2 mRNA levels than controls. When exposed to cold, CR rats experienced a transient decline in body temperature, but the values were reestablished after $24 \mathrm{~h}$, despite having lower UCP1 levels than controls.

CONCLUSIONS: These results suggest that BAT thermogenic capacity is diminished in CR animals, involving impaired BAT sympatheticinnervation and thyroid hormone signaling. These alterations make animalsmore sensitive to coldand maycontribute to long-term outcomes of gestational calorie restriction in promoting obesity and related metabolic alterations.

International Journal of Obesity (2015) xxxxxxxxxxxx

Keywords: calorie restriction; thermogenesis; brown adipose tissue; sympathetic innervation; thyroid hormones; cold response

\section{INTRODUCTION}

Nutritional environment during sensitive periods of early development is thought to program later body weight. The hypothesis of fetal origins of adult diseases, proposed by Barker and collaborators, ${ }^{1}$ suggests that poor fetal nutrition leads to metabolic adaptations programming subjects to be more sensitive to obesity development under a positive nutritional environment. The Dutch Famine epidemiological study showed that the incidence of obesity was higher in men whose mothers underwent malnutrition during the first 6 months of pregnancy due to the acute famine suffered in western Holland during World War II. ${ }^{2}$ In animal models, severe (30 to 50\%) maternal calorie restriction during gestation has been associated to obesity predisposition and other related alterations, such as insulin resistance. ${ }^{3-6}$ More recently, less severe maternal calorie restriction during gestation (20\%) has also been described to program lasting detrimental effects in offspring, but in a sex-dependent manner: effects on body weight maintenance were particularly found in males, although both genders displayed lower insulin and leptin sensitivity accompanied by hyperphagia. ${ }^{7}$ We have described some mechanisms that may account for the effects on food intake $^{8}$ and white adipose tissue (WAT), ${ }^{9}$ but the potential involvement of brown adipose tissue (BAT) has not been explored.

Early programming of adipose depots has been described to be determinant for the later functionality of the tissue. ${ }^{10,11}$ In mammals, besides WAT, there are small amounts of BAT, which is the main site of adaptive thermogenesis, providing extra heat in hibernating animals, newborns and cold-exposed mammals. ${ }^{12}$ Brown adipocytes, unlike the white ones, are characterized by multilocular lipid droplets and a greater amount of mitochondria that express uncoupling protein 1 (UCP1). ${ }^{13,14} \mathrm{UCP} 1$ uncouples the rates of substrate oxidation and ATP production by favoring a loss of protons and energy release in the form of heat. ${ }^{15}$ In addition, BAT is also important in the control of energy balance as it is activated in response to diet. ${ }^{16}$ Therefore, activation of BAT thermogenesis represents a mechanism that enables dissipating, in a regulated manner, part of the energy from food as heat instead of accumulating it as fat. The recent recognition of BAT presence and function in adult humans has promoted a renewed

\footnotetext{
${ }^{1}$ Laboratory of Molecular Biology, Nutrition and Biotechnology (Nutrigenomics), University of the Balearic Islands and CIBER de Fisiopatología de la Obesidad y Nutrición (CIBEROBN), Palma de Mallorca, Spain and ${ }^{2}$ Department of Nutrition and Food Sciences, Faculty of Biology, University of Barcelona and CIBER de Fisiopatología de la Obesidad y Nutrición (CIBEROBN), Barcelona, Spain. Correspondence: Professor A Palou, Laboratory of Molecular Biology, Nutrition and Biotechnology (Nutrigenomics), University of the Balearic Islands, Campus de la Cra. Valldemossa Km 7.5, Balearic Islands, 07122 Palma de Mallorca, Spain.

E-mail: andreu.palou@uib.es

Received 13 January 2014; revised 10 March 2014; accepted 22 March 2014; accepted article preview online 3 April 2014;
} 
interest in BAT thermogenesis as a possible target for stimulation of energy expenditure to help mitigate increased body fat storage. ${ }^{17}$

The sympathetic innervation of BAT is the most significant physiological effector of the thermogenic process. This is triggered by the release of norepinephrine (NE) that turn on a cascade of intracellular events ending in activation of UCP1, together with other processes, including triglyceride (TG) mobilization, thus providing the fatty acids necessary for UCP1 activation. ${ }^{15,16}$ Thyroid hormones also have a key role in the activation of adaptive thermogenesis. In concrete, triiodothyronine (T3) is necessary for the full expression of UCP1 and amplifies the adrenergic stimulation of UCP1 mRNA expression in cold-exposed rats. ${ }^{18}$ T3 also increases the adrenergic stimulation of BAT deiodinase iodothyronine type II (DIO2) ${ }^{19}$ a selenoenzyme that locally produces T3, via 5'-deiodination of thyroxine (T4).

Given the contribution of BAT to energy homeostasis and its potential interest as a target for obesity therapy, here we aimed to assess whether impaired BAT thermogenesis capacity could also contribute to adverse outcomes of maternal calorie restriction during gestation in relation to a higher propensity to develop overweight and related metabolic alterations, and, if so, to ascertain which mechanisms could be responsible for this alteration.

\section{MATERIALS AND METHODS}

The animal protocol followed in this study was reviewed and approved by the Bioethical Committee of our University and guidelines for the use and care of laboratory animals of the University were followed.

\section{Study 1}

Animals and experimental design. The study was performed in 25-day-old male and female rats from 29 different litters, including the offspring of control and $20 \%$ calorie-restricted rats during the first part of gestation (CR), as previously described. ${ }^{7}$ All rats were housed under controlled temperature $\left(22^{\circ} \mathrm{C}\right)$ and a 12 -h light-dark cycle (light on from 0800 to 2000 hours), and had unlimited access to tap water and standard chow $\operatorname{diet}\left(3 \mathrm{kcal} \mathrm{g}^{-1}\right.$, with $8 \%$ calories from fat; Panlab, Barcelona, Spain) unless mentioned otherwise. Briefly, virgin female Wistar rats weighing between $200 \mathrm{~g}$ and $250 \mathrm{~g}$ were mated with male rats (Charles River Laboratories, Barcelona, Spain). Day of conception (day 0 of pregnancy) was determined by examination of vaginal smears for the presence of sperm, and then female rats were singlecaged. Pregnantrats were dividedinto two groups: one with free access to standard chow diet $(n=13)$, and the other one underwent $20 \%$ restriction of calorie intake from day 1 to day 12 of pregnancy $(n=16)$. Calorie restriction was performed by offering each dam, at the beginning of the light phase, a daily amount of food corresponding to $80 \%$ of the calories that should be eaten according to body weight. This amount was calculated considering the calories consumed daily by their control animals under ad libitum feeding conditions. After the calorie restriction period, rats were allowed to eat ad libitum. On day 1 after delivery, excess pups in each litter were removed to keep 10 pups per dam (five males and five females, when possible). Weaning was conducted on postnatal day 21.

At the age of 24 days, body weight of pups was recorded. On day 25 of life and during the first $2 \mathrm{~h}$ of the beginning of the light cycle, one group of animals from control dams and from calorie-restricted dams was killed by decapitation after 12-h fasting ( $n=6-8$ animals per group) and a second group of rats was killed under ad libitum feeding conditions ( $n=5-8$ animals per group). Blood samples were collected from the neck in heparinized containers. Plasmawas obtained by centrifugation of blood at $1000 \mathrm{~g}$ for $10 \mathrm{~min}$ and stored at $-20^{\circ} \mathrm{C}$ until analysis. Interscapular BAT was rapidly removed, weighed and frozen in liquid nitrogen and stored at $-80^{\circ} \mathrm{C}$ until subsequent studies. Tissue analyses were conducted in BAT of animals under basal (12-h fasting) conditions.

Measurement of circulating parameters. Blood glucose concentration was measured by Accu-Chek Glucometer (Roche Diagnostics, Barcelona, Spain). Insulin concentration was determined using ultrasensitive rat insulin ELISA (enzyme-linked immunosorbentassay) kit(MercodiaAB, Uppsala, Sweden), and leptin concentration was measured using ELISA kit Quantikine Mouse Leptin Inmunoassay (R\&D Systems, Minneapolis, MN, USA). Thyroid-stimulating hormone (TSH), triiodothyronine (T3) and thyroxine (T4) circulating levels were analyzed by using the commecial ELISA kits EIA-1780 (T3), EIA-1781 (T4) and EIA-5296 (TSH) (DRG, Marburg, Germany).

Western blot analysis. The amount of UCP1 and tyrosine hydroxylase (TyrOH) in BAT of control and CR rats was determined by western blot. Tissue was homogenized at $4{ }^{\circ} \mathrm{C}$ in 1:10 (w:v) of phosphate buffer saline. The homogenate was centrifuged at $500 \mathrm{~g}$ for $10 \mathrm{~min}$ at $4{ }^{\circ} \mathrm{C}$ and the supernatant was used for protein analysis. Total protein content was measured by the method of Bradford. ${ }^{20}$ For analysis, $40 \mu \mathrm{g}$ of total protein was solubilized and boiled for $3 \mathrm{~min}$ in Laemmli sample buffer containing $5 \%$ 2-beta-mercaptoethanol. Then, total protein was fractionated by SDS-polyacrylamide gel electrophoresis ( $10 \%$ polyacrylamide), and electrotransferred onto nitrocellulose membrane (Bio-Rad, Madrid, Spain). Black amide B10 staining provided visual evidence for correct loading and blotting of proteins. After blocking, the membrane was incubated with the primary rabbit polyclonal anti-UCP1 (GeneTex Inc., Irvine, CA, USA) or antiTyrOH (Santa Cruz Biotechnology Inc., Dallas, TX, USA), and then with the infrared-dyed secondary anti-IgG antibody (LI-COR Biosciences, Lincoln, NE, USA) diluted 1:25 000. For infrared detection, membranes were scanned in Odyssey Infrared Imaging System (LI-COR Biosciences), and the bands were quantified using the analysis software provided.

RNA extraction. Total RNA was extracted from BAT by EZNA TOTAL RNA kit I (Omega Bio-Tek Inc., Norcross, GA, USA) according to the manufacturer's instructions. Isolated RNA was quantified using the NanoDrop ND-1000 spectrophotometer (NadroDrop Technologies Inc., Wilmington, DE, USA) and its integrity confirmed using $1 \%$ agarose gel electrophoresis.

Real-time quantitative PCR (RT-qPCR) analysis. Real-time PCR was used to measure mRNA expression levels of DIO2, lipoprotein lipase (LPL) and CPT1 beta in BAT. An amount $0.25 \mu \mathrm{g}$ of total RNA (in a final volume of $5 \mu \mathrm{l}$ ) was denatured at $65^{\circ} \mathrm{C}$ for $10 \mathrm{~min}$ and then reverse transcribed to cDNA using MuLV reverse transcriptase (Applied Biosystems, Madrid, Spain) at $20^{\circ} \mathrm{C}$ for $15 \mathrm{~min}, 42^{\circ} \mathrm{C}$ for $30 \mathrm{~min}$, with a final step of $5 \mathrm{~min}$ at $95^{\circ} \mathrm{C}$ in an Applied Biosystems 2720 Thermal Cycler (Applied Biosystems). Each PCR was performed from diluted cDNA template, forward and reverse primers (1 $\mu$ meach), and Power SYBER Green PCR Master Mix (Applied Biosystems). Real-time PCR was performed using the Applied Biosystems StepOnePlusTM Real-Time PCR Systems (Applied Biosystems) with the following profile: $10 \mathrm{~min}$ at $95^{\circ} \mathrm{C}$, followed by a total of 40 two-temperature cycles $(15 \mathrm{~s}$ at $95^{\circ} \mathrm{C}$ and $1 \mathrm{~min}$ at $60^{\circ} \mathrm{C}$ ). To verify the purity of the products, a melting curve was produced after each run according to the manufacturer's instructions. The Ct (threshold cycle) was calculated by the instrument's software (StepOne Software v2.1) and the relative expression of each mRNA was calculated as a percentage of male control rats, using the $2^{-\Delta \Delta C t}$ method; ${ }^{21}$ beta-actin and GDI1 were used as reference genes. Sequences of primers (obtained from Sigma, Madrid, Spain) were: betaactin, forward: 5'-TACAGCTTCACCACCACAGC-3', reverse: 5'-TCTCCAGG GAGGAAGAGGAT-3'; GDI1, forward: 5'-CCGCACAAGGCAAATACATC-3', reverse: 5'-GACTCTCTGAACCGTCATCAA-3'; DIO2, forward: 5'-TTCTCCAAC TGCCTCTTCCT-3', reverse: 5'-CAGGTCGCTGAACCAAAGT-3'; LPL, forward: 5'-TATGGCACAGTGGCTGAAAG-3', reverse: 5'-CTGACCAGCGGAAGTAGGAG-3'; CPT1, forward: 5'-GCAAACTGGACCGAGAAGAG-3', reverse: 5'-CCTTGAAGA AGCGACCTTTG-3. Stable expression of reference genes was checked. The maximum difference between $\mathrm{Ct}$ averages of the different groups was 0.400 (for beta-actin) and 0.266 (for GDI1).

\section{Study 2}

In view of the results obtained in the first study, in a second step we analyzed the response to cold exposure of controls and CR rats. For this, a new cohort of controls and calorie-restricted rats from eight different litters (four controls and four calorie-restricted dams) was obtained as described above. At the age of 24 days, controls and CR rats were exposed to cold $\left(6^{\circ} \mathrm{C}\right)$ for $24 \mathrm{~h}$ and rectal temperatures were recorded at specific time points: $0,1,2$, 4 and $24 \mathrm{~h}$. Temperatures were recorded from $15-18$ rats per group. On day 25, after the 24-h cold exposure period, a set of animals from each group (control and CR) was killed ( $n=8-10$ rats per group) and interscapular BAT was rapidly collected for western blot analyses of UCP1. Western blot was performed as described for study 1 , but using $10 \mu \mathrm{g}$ of protein. 
Statistical analysis

Data are expressed as means \pm s.e.m. Two-way, three-way or repeated measures analysis of variance (ANOVA) with the factors of calorie restriction $(R)$, sex $(S)$, fasting $(F)$ or cold exposure $(C)$ was performed to assess statistical significances. Individual means were compared with a Student's $t$-test or Paired $t$-test. The analyses were performed with SPSS for Windows (SPSS, Chicago, IL, USA). Threshold of significance was defined at $P$ o 0.05 .

\section{RESULTS}

Study 1

As previously described in another group of animals, ${ }^{8}$ control and $\mathrm{CR}$ animals exhibited no significant differences in their body weight at the age of 24 days, or in the weight of BAT at sacrifice (data not shown).

Blood parameters are shown in Table 1. Twelve hours of fasting caused a significant decreased in glucose, insulin and leptin in all groups of animals ( $P \mathbf{O} 0.05$, two-way ANOVA). No significant differences were found in glucose, insulin, leptin, TSH or T4 circulating levels due to maternal calorie restriction during gestation. However, CR rats showed lower plasma levels of the T3 hormone ( $P$ O 0.05, two-way ANOVA), with the differences being more marked and significant by Student's $t$-test $(P$ 00.05) only in males.

Figure 1a shows specific protein levels of UCP1 in BAT of male and female control and $\mathrm{CR}$ rats. $\mathrm{CR}$ animals revealed lower UCP1 levels than controls ( $P$ O 0.05 , two-way ANOVA).

Specific protein levels of TyrOH in BAT are shown in Figure 1b. $\mathrm{CR}$ animals also showed lower TyrOH protein levels than their controls ( $P$ O 0.05, two-way ANOVA), although the decrease was more pronounced and only significant by Student's $t$-test ( $P$ o0.05) in CR males.

Gene expression levels of DIO2 are shown in Figure 1c. Notably, a sex-dependent effect of maternal calorie restriction during gestation was found (interactive effect between calorie restriction

\begin{tabular}{|c|c|c|c|c|c|}
\hline & \multicolumn{2}{|c|}{ Males } & \multicolumn{2}{|c|}{ Females } & \multirow[t]{2}{*}{ ANOVA } \\
\hline & Control & $C R$ & Control & $C R$ & \\
\hline \multicolumn{6}{|c|}{ Glucose $\left(m g d l^{-1}\right)$} \\
\hline Fed & $148 \pm 4(8)$ & $145 \pm 2(7)$ & $149 \pm 4(7)$ & $148 \pm 5(7)$ & $\mathrm{F}$ \\
\hline Fasting & $76 \pm 4(8)$ & $78 \pm 5(6)$ & $64 \pm 3(6)$ & $59 \pm 5(6)$ & \\
\hline \multicolumn{6}{|c|}{ Insulin (ng |-1) } \\
\hline Fed & $170 \pm 24(8)$ & $204 \pm 33(7)$ & $248 \pm 41(7)$ & $240 \pm 49(7)$ & $\mathrm{F}$ \\
\hline Fasting & $40 \pm 6(6)$ & $48 \pm 8(6)$ & $52 \pm 5(6)$ & $47 \pm 9(6)$ & \\
\hline \multicolumn{6}{|c|}{ Leptin $\left(n g l^{-1}\right)$} \\
\hline Fed & $2206 \pm 170(8)$ & $1798 \pm 121(7)$ & $1689 \pm 241(7)$ & $1461 \pm 145(7)$ & $\mathrm{F}$ \\
\hline Fasting & $40 \pm 24(6)$ & $17 \pm 8(6)$ & $27 \pm 13(6)$ & $78 \pm 51(6)$ & \\
\hline \multicolumn{6}{|c|}{$T S H\left(n g m l^{-1}\right)$} \\
\hline \multicolumn{6}{|c|}{ Fed $\quad 1.18 \pm 0.07(8) 1.14 \pm 0.08$ (7) $1.47 \pm 0.23(7) 1.23 \pm 0.12(5)$} \\
\hline \multicolumn{6}{|c|}{ T3 $\left(n g m l^{-1}\right)$} \\
\hline \multicolumn{6}{|c|}{ Fed $\quad 5.64 \pm 0.37(8) 4.13 \pm 0.45^{a}$ (7) $5.63 \pm 0.58(7) 4.54 \pm 0.35(5) R$} \\
\hline \\
\hline \multicolumn{6}{|c|}{ Fed $\quad 45.4 \pm 2.3(8) 49.4 \pm 1.6(7) 45.9 \pm 0.6(7) 46.3 \pm 2.4(5)$} \\
\hline \multicolumn{6}{|c|}{$\begin{array}{l}\text { Blood levels of glucose, insulin and leptin under fed and fasting conditions } \\
\text { and of thyroid-stimulating hormone (TSH), triiodothyronine (T3) and } \\
\text { thyroxine (T4) under fed conditions of male and female offspring from } \\
\text { controls and calorie-restricted dams during gestation (CR) at the age of } 25 \\
\text { days. Data are mean } \pm \text { s.e.m. The number of animals per group is indicated } \\
\text { in brackets. Statistics: F, effect of fasting ( } P \text { o0.05, three-way analysis of } \\
\text { variance (ANOVA)); R, effect of calorie restriction ( } P \text { o0.05, two-way } \\
\text { ANOVA). a CR versus control ( } P \text { o0.05; Student's } t \text {-test). }\end{array}$} \\
\hline
\end{tabular}
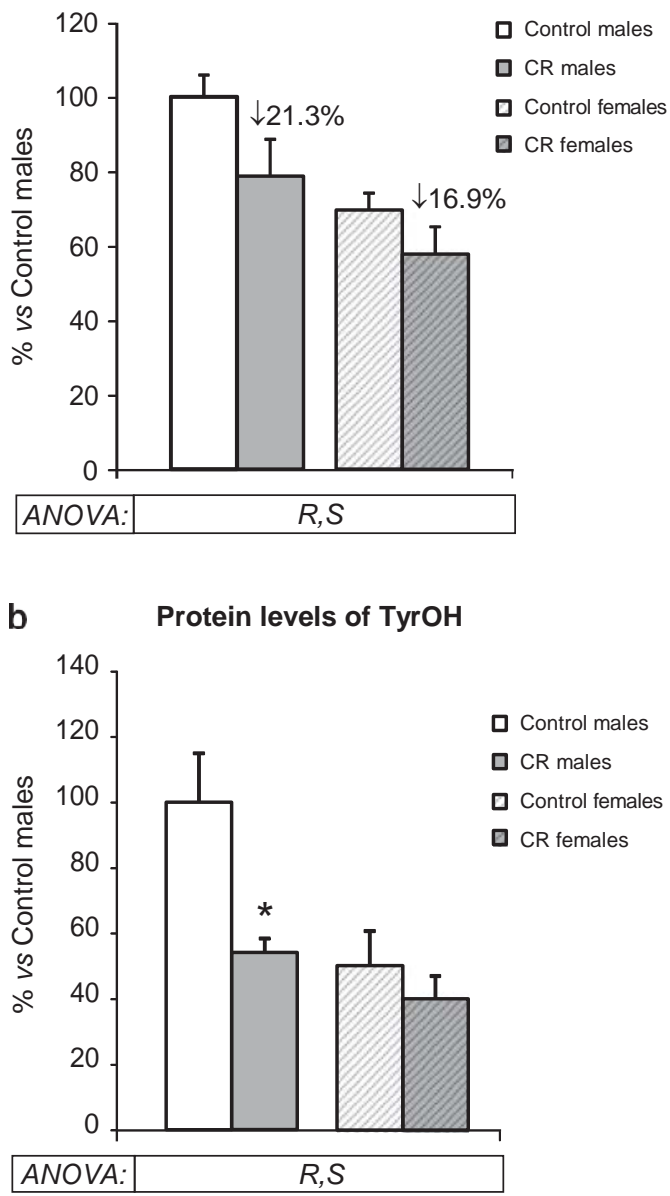

C mRNA levels of DIO2

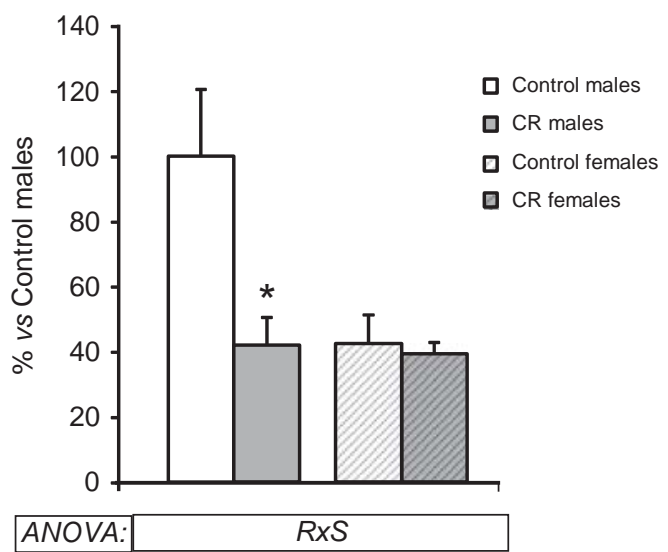

Figure 1. Specific abundance of uncoupling protein 1 (UCP1) (a) and of tyrosine hydroxylase protein (TyrOH) (b), and mRNA levels of deiodinase iodothyronine type II (DIO2) (C), in brown adipose tissue of 25-day-old male and female offspring from control and calorierestricted dams during gestation (CR). Data are expressed as a percentage of the mean value of control male rats. Data are mean \pm s.e.m. $(n=6-8)$. Percentages of decrease of UCP1 levels in CR rats with respect to controls are indicated. Statistics: $R$, effect of calorie restriction; $S$, effect of sex; and RxS, interactive effect between calorie restriction and sex ( $P 00.05$, two-way ANOVA). *, CR versus control ( $P$ o0.05; Student's $t$-test). 
and sex, $P$ O 0.05, two-way ANOVA); whereas CR males showed lower DIO2 mRNA levels compared with controls $(P \mathbf{O} 0.05$, Student's $t$-test), no significant differences were found among females.

Figure 2 shows mRNA levels of LPL and CPT1 in control and CR rats. Expression levels of both genes were lower in CR animals compared with controls ( $P$ O 0.05, two-way ANOVA). The decrease in LPL mRNA expression levels was more marked and only significant by Student's $t$-test $(P$ o0.05) in CRmales.

\section{Study 2}

Table 2 shows the weight-related parameters of controls and CR animals belonging to the second study. In this study, both male and female $\mathrm{CR}$ animals already showed higher body weight than their controls at the age of 24 days (PO0.05, two-way ANOVA). The reason for the difference from Study 1 is not known; this could be potentially attributed to particular characteristics of the different cohorts of animals, which could have caused a more prompt effect on body weight gain of CR animals than in Study 1. Of note, no significant differences were found at birth between controls and CR rats in either study (data notshown). In this study, it was shown that both male and female control animals increased their body weight over the 24-h cold exposure period, whereas body weight of $\mathrm{CR}$ animals remained practically unchanged (PO 0.05, two-way ANOVA). No significant differences were found in the weight of BAT between control and CR animals at sacrifice.

Figure 3 shows body temperature during cold exposure in control and CR rats. In males (Figure 3a), cold exposure caused a

mRNA levels of LPL and CPT1

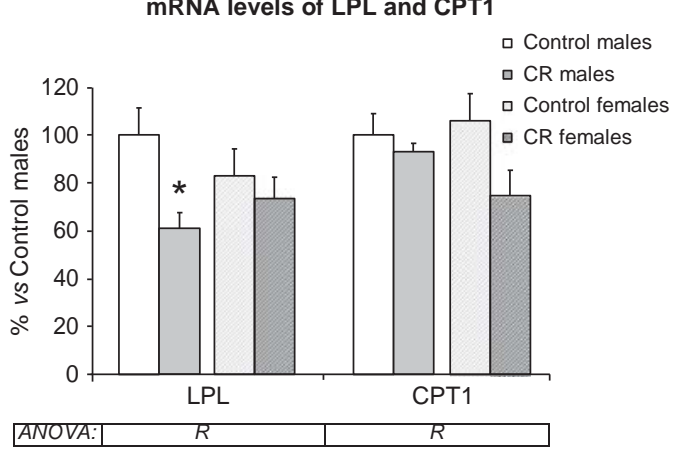

Figure 2. mRNA levels of lipoprotein lipase (LPL) and carnitine palmitoyltransferase 1 (CPT1) beta in brown adipose tissue of 25 day-old male and female offspring from control and calorierestricted dams during gestation (CR). Data are expressed as a percentage of the mean value of control male rats. Data are mean \pm s.e.m. $(n=6-8)$. Statistics: $R$, effect of calorie restriction $\left(P\right.$ 00.05, two-way ANOVA). ${ }^{*}, \mathrm{CR}$ versus control ( $P$ 00.05; Student's $t$-test). significant reduction in body temperature in the CR group after 1 and $2 \mathrm{~h}$, but they partially recovered their normal temperature after $4 \mathrm{~h}$ and totally after $24 \mathrm{~h}$ ( $P$ O 0.05 , Paired $t$-test); in contrast, control rats maintained their temperature throughout the whole period studied. After $2 \mathrm{~h}$ of cold exposure, CR male animals showed a trend to lower temperature compared with controls ( $P=0.061$, Student's $t$-test), and an interactive effect between

Body temperature response during 24-h of cold exposure
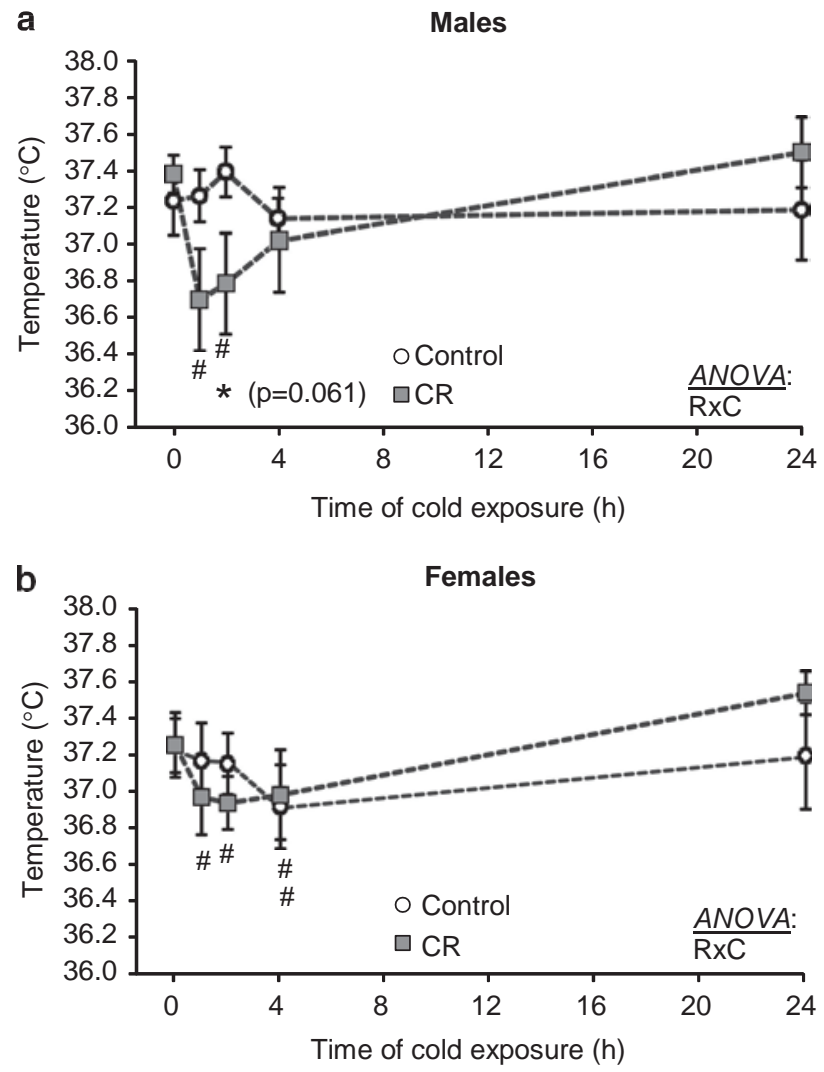

Figure 3. Body temperature of male (a) and female (b) offspring from control and calorie-restricted dams during gestation (CR) over 24$\mathrm{h}$ cold exposure (from day 24 to day 25 of life), measured at different time points: before cold exposure (T0) and after 1- (T1), 2- (T2), 4- (T4) and 24-h (T24). Data are mean \pm s.e.m. $(n=11-18)$. Statistics: $R \times C$ interactive effect between calorie restriction and the time response to cold ( $P$ 00.05, ANOVA repeated measures). \#, T1 or T2 or T4 or T24 versus T0 ( $P$ 00.05, Paired $t$-test); and $*$, CR versus control $(P=0.061$, Student's $t$-test).

Table 2. Study 2: Weight-related parameters

\begin{tabular}{|c|c|c|c|c|c|}
\hline & \multicolumn{2}{|c|}{ Males } & \multicolumn{2}{|c|}{ Females } & \multirow[t]{2}{*}{ ANOVA } \\
\hline & Control (8) & $C R(10)$ & Control (8) & $C R(10)$ & \\
\hline Body weight (g) & $58.7 \pm 2.0$ & $65.5 \pm 1.1^{\mathrm{a}}$ & $58.1 \pm 1.7$ & $61.9 \pm 1.2$ & $\mathrm{R}$ \\
\hline Body weight gain (g) & $1.66 \pm 0.29$ & $0.367 \pm 0.349^{a}$ & $1.41 \pm 0.64$ & $-0.446 \pm 0.613$ & $\mathrm{R}$ \\
\hline Body weight gain (\%) & $2.72 \pm 0.38$ & $0.579 \pm 0.532^{\mathrm{a}}$ & $2.53 \pm 1.16$ & $-0.636 \pm 0.954^{a}$ & $\mathrm{R}$ \\
\hline BAT weight (mg) & $159 \pm 6$ & $175 \pm 10$ & $155 \pm 6$ & $165 \pm 7$ & \\
\hline
\end{tabular}

Body weight (at the age of 24 days, before cold exposure), body weight gain during the 24-h period of cold exposure and weight of BAT at sacrifice (day 25) of male and female offspring from control and calorie-restricted dams during gestation (CR). Data are mean \pm s.e.m. The number of animals per group is

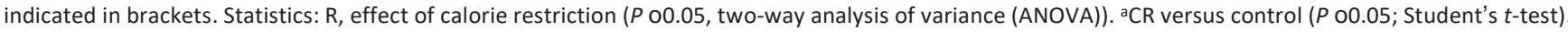


maternal calorie restriction and body temperature over time during cold exposure ( $P$ O 0.05 , ANOVA repeated measures) was found. A different response to cold was also found between control and CR female animals (interactive effect between maternal calorie restriction and body temperature over time, $P$ O 0.05, ANOVA repeated measures) (Figure $3 b$ ). CR female rats showed a decrease in their body temperature during the first $4 \mathrm{~h}$ of cold exposure ( $P \mathbf{O} 0.05$, Paired $t$-test), although they managed to recover it at $24 \mathrm{~h}$. On the other hand, control females maintained their temperature during the first $2 \mathrm{~h}$, but displayed a significant reduction in their temperature at $4 \mathrm{~h}(P$ O 0.05, Paired $t$-test), which was reestablished after $24 \mathrm{~h}$.

Specific UCP1 protein levels in BAT of control and CR animals after 24-h cold exposure are shown in Figure 4. Under these conditions, both male and female $\mathrm{CR}$ rats also revealed lower UCP1 levels than controls ( $P$ O 0.05, two-way ANOVA), with the differences being more marked than those observed at $22^{\circ} \mathrm{C}$.

\section{DISCUSSION}

The presence and activity of BAT in adult humans has reopened the interest for this tissue as a target for obesity therapy and other metabolic-related pathologies, such as insulin resistance and type 2 diabetes. ${ }^{12,22}$ Research aimed at ascertaining which factors and conditions during early stages of life may program BAT function and activity, and hence contribute to later effects on the capacity to maintain body weight, is becoming of great interest.

We previously showed that moderate $(20 \%)$ maternal calorie restriction during gestation programs lasting detrimental effects in offspring, particularly obesity, insulin resistance and alterations in other risk factors of cardiovascular disease and diabetes. ${ }^{7}$ Potential mechanisms involved in the programming effects of this nutritional condition during pregnancy still remain to be fully elucidated. Here we show for the first time that $20 \%$ maternal calorie restriction during the first part of gestation results in the offspring in a significant reduction of specific UCP1 protein levels in BAT: $21.3 \%$ and $16.9 \%$ in males and females, respectively, compared with their controls. Although we have not directly measured energy expenditure or BAT functioning, the reduction

\section{Protein levels of UCP1 in BAT}

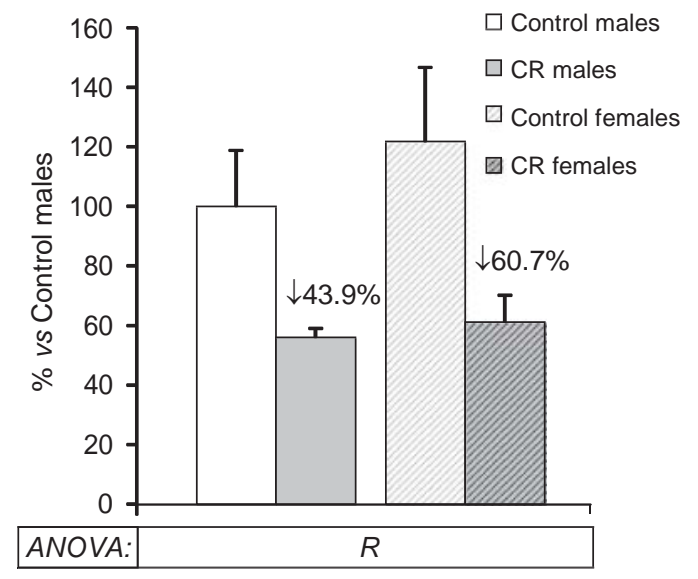

Figure 4. Specific abundance of uncoupling protein 1 (UCP1) in brown adipose tissue of 25-day-old male and female offspring from control and calorie-restricted dams during gestation (CR) after 24-h cold exposure. Data are expressed as a percentage of the mean value of control rats. Data are mean \pm s.e.m. $(n=6)$. Percentages of decrease of UCP1 levels in CR rats with respect to controls are indicated. Statistics: $\mathrm{R}$, effect of calorie restriction ( $P$ 00.05, two-way ANOVA). of UCP1 levels might be interpreted as a decreased thermogenic capacity, although this is an indirect measure. The lower burning capacity of BAT could contribute, at least in part, to the greater predisposition to fat accumulation and other metabolic alterations that CR animals suffer, particularly males, in adulthood. ${ }^{7}$ The acute activity of BAT, such as heat production, and the recruitment process in the tissue are under the control of NE released from sympathetic nerves. ${ }^{15,23}$ To ascertain whether the reduced thermogenic capacity of $\mathrm{CR}$ rats could be due to an impairment in sympathetic innervation, the sympathetic drive was estimated by measuring $\mathrm{TyrOH}$ protein levels, the rate-limiting enzyme in catecholamine synthesis. ${ }^{24}$ Protein content measurement of this enzyme has been described to be a good estimation of sympathetic innervations. ${ }^{25}$ Interestingly, we found that $\mathrm{CR}$ animals exhibited lower TyrOH levels in BAT; the decrease occurred in both males and females, although it was only significant by Student's $t$-test in males (45.9\% decrease compared with their respective controls). Notably, a significant positive correlation between UCP1 and TyrOH proteinlevelsin BAT was also found $(P=0.040)$. This is in agreement with the relationship between sympathetic innervation and UCP1 production in BAT, therefore suggesting that the reduced thermogenic capacity could be due, at least in part, to impaired sympathetic signaling. The alterations in sympathetic innervation occurring in CR animals do not seem to be exclusive of BAT. We previously described, in a different group of animals, that male offspring of moderate CR rats during gestation, but not females, showed lower iWAT sympathetic innervations. ${ }^{9}$ Hence, it could be speculated that an impairment in WAT and BAT sympathetic innervation may contribute to the detrimental effects of maternal calorie restriction during gestation in offspring; sex-dependent differences in the occurrence and magnitude of the alteration may in turn account for the previously described sex-dependent outcomes in adult body weight and adiposity. ${ }^{26}$

The lower burning capacity of BAT occurring in CR animals was found to be associated with lower mRNA expression levels of genes related to fuel supply and fatty acid oxidation in this tissue. CR animals displayed lower LPL and CPT1 mRNA levels, compared withcontrols. LPLactivityin BAT increases the supplyoffattyacids from circulating lipoproteins for metabolic needs and maintenance of thermogenesis. When TG stored in BAT are not enough to maintain heat production, extra lipids have to be imported into the tissue; LPL is the enzyme responsible for the delivery of fatty acids from circulating lipoproteins. ${ }^{23}$ Unlike what occurs in WAT, LPL activity and expression in BAT is under the control of NE. ${ }^{27}$ Thus, the decrease in mRNA levels of LPL found in CR animals, particularly in males, may be reflecting the alterations in sympathetic innervation occurring in these animals, which may in turn lead to a lower fuel supply for thermogenic activity. Although LPL present in BAT has generally received less attention compared with that present in the other peripheral tissues, such as WAT and skeletal muscle, changes in the amount or activity of this enzyme in BAT may also contribute to plasma clearance of TG. ${ }^{28}$ In this regard, we previously described that both male and female young adult CR animals displayed higher circulating TG levels than their controls. ${ }^{7}$ Meanwhile, CPT1 catalyzes the limiting rate for mitochondrial $\beta$-oxidation, constituting the major pathway whereby fatty acids are oxidized to generate energy. ${ }^{29}$ The observed reduced CPT1 mRNA expression levels in BAT of CR rats amount to a likely metabolic profile in BAT where a lower capacity for fatty acid uptake accompanies a limited fatty acid oxidation capacity.

Thyroid hormones also have a fundamental role in BAT activity. ${ }^{30}$ The control of thermogenesis by NE is under the action of the thyroid hormones T3 and T4. T4 is converted to the active form T3 by the deiodinase iodothyronine enzymes. ${ }^{31}$ Thyroid hormones interact with the sympathetic nervous system, being critical for the full activation of NE signaling cascade and full 
thermogenesis activation in BAT. ${ }^{18}$ In fact, the disability of hypothyroid rodents to activate BAT thermogenesis by NE can be solved by the administration of $\mathrm{T} 3$ or $\mathrm{T} 4 .^{32,33} \mathrm{DIO} 2$ is also an essential component in the thyroid-sympathetic synergism required for thermal homeostasis in small mammals. ${ }^{34}$ The main role of DIO2 is to regulate intracellular T3 concentration, its accessibility to the nucleus and the saturation of the nuclear T3 receptor in target tissues. ${ }^{31}$ Transgenic mice with a disruption of DIO2 exhibit dramatically impaired thermogenesis in BAT and a greater predisposition to diet-induced obesity, in spite of having normal plasma T3 concentration. ${ }^{34,35}$

Therefore, the sympathetic nervous system is essential for the activation of BAT thermogenesis, although its responsiveness is dependent on the concentration of T3 in the tissue, which in turn depends on the DIO2 activity that is stimulated by NE. ${ }^{18}$ In the present study, we found that CR animals, and particularly males, presented lower plasma T3 levels and lower BAT DIO2 expression levels. The reduced concentration of the active form of thyroid hormones is not only in agreement with the greater propensity to develop overweight of these animals, as hypothyroidism has been related to higher fat accumulation, ${ }^{36}$ but also with reduced UCP1 production. It could be propounded that the decreased T3 plasma levels occurring in CR animals, togetherwith the lower expression levels of DIO2 in BAT, may impair NEsignaling in this tissue, which is impaired per se due to maternal conditions during gestation, and altogether contribute to the reduced burning capacity of $\mathrm{CR}$ animals. It is noteworthy that the reduction in DIO2 mRNA levels was only found in CR males, and they also displayed a more dramatic decrease in sympathetic drive to BAT in comparison with females. This sex-dependent response is in agreement with the major reduction in T3 plasma levels occurring in males and their proneness to fat accumulation later in life, as previously described. ${ }^{7}$

All in all, the results obtained so far suggest that CR animals have an imprinted impaired BAT thermogenic capacity compared with controls. To ascertain whether this situation occurring in $\mathrm{CR}$ animals could affect their thermoregulation capacity, we evaluated body temperature during a $24 \mathrm{~h}$ period of cold exposure (Study 2). Interestingly, CR animals, both males and females, showed a deficient capacity to maintain body temperature during the first hours of cold exposure, but they managed to restore their normal temperature after $4 \mathrm{~h}$ (at least partially, in the case of males) or $24 \mathrm{~h}$ in the case of females. Control rats, particularly males, presented a better capacity to maintain body temperature during the period studied. These results suggest that thermoregulatory capacity is diminished in CR rats, although they can manage to maintain body temperature after an acclimatizing period, at least during short periods of cold exposure as studied here.

Increased UCP1 production does not seem to be responsible for the recovery of normal body temperature after $24 \mathrm{~h}$ of cold exposure. CR animals showed lower UCP1 levels in BAT than controls. The difference between control and CR animals was more exacerbated (decreases of $43.9 \%$ and $60.7 \%$ in male and female animals, respectively) compared with what was found at $22{ }^{\circ} \mathrm{C}$ in Study 1 . This suggests that cold-induced BAT thermogenesis is also impaired in CR rats. Maintenance of body temperature in $\mathrm{CR}$ animals found at $24 \mathrm{~h}$ of cold exposure may be tentatively attributed, at least in part, to a greater activation of shivering thermogenesis. It is known that when unacclimatized animals are placed in a cold environment, they acutely defend their body temperature by activation of both BAT thermogenesis (also referred as non-shivering thermogenesis) and shivering thermogenesis in skeletal muscle. It has been estimated that the latter may provide around one-third of the total heat production during cold exposure, being very important in the maintenance of homeothermy. ${ }^{37}$ However, this form of thermogenesis consumes large amounts of energy and interferes with normal activity. ${ }^{18}$
In agreement with this, body weight gain in CR animals during the 24-h period of cold exposure was negligible or even tended to decrease, and was in any case significantly lower compared with that of controls. This suggests that CR animals exhibited greater activation of the shivering thermogenesis to counterbalance the deficient activation of BAT (non-shivering) thermogenesis. Alternative explanations could also be possible. Maintenance of body temperature in CR animals could be achieved by increased voluntary locomotor activity to combat cold exposure, and/or by increased metabolic rate or futile cycling in other organs than BAT, which is supported by the absence of an increase in body weight during the 24-h cold exposure. Hence, although the exact contributing mechanism is not known, results suggest that the difference between short-term ( $04 \mathrm{~h}$ ) and long-term (24 h) response to cold observed between control and $\mathrm{CR}$ animals might involve BAT and non-BAT effects. All in all, our results support the fact that $\mathrm{CR}$ animals have defective BAT thermogenesis, associated with a delayed thermal response to cold and to inefficient thermoregulation, and hence probably making these animals more sensitive to cold. Whether these results can be extrapolated to human is not known, but in view of the described presence of active BAT in humans and its relation with obesity, ${ }^{22}$ present findings may give some cues for the early programming mechanisms of later diseases, particularly regarding energy dysbalance in adulthood, and hence merits to be investigated.

In summary, it can be outlined that moderate (20\%) maternal calorie restriction during the first 12 days of pregnancy programs offspring for lower BAT thermogenic capacity at the juvenile age of 25 days, which may determine a greater sensitivity to cold and contribute to the higher propensity for fat accumulation and other metabolic alterations later in life. Both impaired sympathetic drive and altered thyroid hormone signaling in BAT, which occur particularly in males, are proposed as mechanisms contributing to the reduced BAT burning capacity. These results pinpoint the relevance of BAT activity programming in the control of later body weight and energy metabolism and also highlight the importance of adequate maternal nutrition during critical periods of development to establish later metabolic health in the offspring, as even moderate interventions may cause a myriad of malprogramming effects.

\section{CONFLICT OF INTEREST}

The authors declare no conflict of interest.

\section{ACKNOWLEDGEMENTS}

The research leading to these results was supported by the Spanish Government (grants AGL2012-33692 to AP and AGL2011-23635 to XR), the European Union's Seventh Framework Programme FP7 2007-2013 under grant agreements n. 244995 (BIOCLAIMS Project) and 278373 (DIABAT Project) to AP, and the Instituto de Salud Carlos III, Centro de Investigación Biomédica en Red Fisiopatología de la Obesidad y Nutrición, CIBERobn. The Laboratory of Molecular Biology, Nutrition, and Biotechnology (Nutrigenomics) is a member of the European Research Network of Excellence NuGO (The European Nutrigenomics Organization, EU Contract: n. FP6-506360). Jadwiga Konieczna is granted with a PhD fellowship entitled 'beca para la formación de personal investigador, en el marco de un programa operativo cofinanciado por el Fondo Social Europeo'.

\section{REFERENCES}

1 Godfrey KM, Barker DJ. Fetal nutrition and adult disease. Am J Clin Nutr 2000; 71(5 Suppl): 1344S-1352S.

2 RavelliGP, Stein ZA, Susser MW. Obesity in young men after famine exposure in utero and early infancy. N Eng/ J Med 1976; 295: 349-353.

3 Anguita RM, Sigulem DM, Sawaya AL. Intrauterine food restriction is associated with obesity in young rats. J Nutr 1993; 123: 1421-1428.

4 Jones AP, Friedman MI. Obesity and adipocyte abnormalities in offspring of rats undernourished during pregnancy. Science 1982; 215: 1518-1519. 
5 Jones AP, Simson EL, Friedman MI. Gestational undernutrition and the development of obesity in rats. J Nutr 1984; 114: 1484-1492.

6 Vickers MH, Breier BH, Cutfield WS, Hofman PL, Gluckman PD. Fetal origins of hyperphagia, obesity, and hypertension and postnatal amplification by hypercaloric nutrition. Am J Physiol Endocrinol Metab 2000; 279: E83-E87.

7 Palou M, Priego T, Sanchez J, Palou A, Pico C. Sexual dimorphism in the lasting effects of moderate caloric restriction during gestation on energy homeostasis in rats is related with fetal programming of insulin and leptin resistance. Nutr Metab (Lond) 2010; 7: 69.

8 Garcia AP, Palou M, Priego T, Sanchez J, Palou A, Pico C. Moderate caloric restriction during gestation results in lower arcuate nucleus NPY- and $\alpha \mathrm{MSH}$ neurons and impairs hypothalamic response to fed/fasting conditions in weaned rats. Diabetes Obes Metab 2010; 12: 403-413.

9 Garcia AP, Palou M, Sanchez J, Priego T, Palou A, Pico C. Moderate caloric restriction during gestation in rats alters adipose tissue sympathetic innervation and later adiposity in offspring. PLoS One 2011; 6: e17313.

10 Priego T, Sanchez J, Garcia AP, Palou A, Pico C. Maternal dietary fat affects milk fatty acid profile and impacts on weight gain and thermogenic capacity of suckling rats. Lipids 2013; 48: 481-495.

11 Symonds ME, Budge H, Perkins AC, Lomax MA. Adipose tissue development-impact of the early life environment. Prog Biophys Mol Biol 2011; 106: 300306.

12 Tam CS, Lecoultre V, Ravussin E. Brown adipose tissue: mechanisms and potential therapeutic targets. Circulation 2012; 125: 2782-2791.

13 Sell H, Deshaies Y, Richard D. The brown adipocyte: update on its metabolic role. Int J Biochem Cell Biol 2004; 36: 2098-2104.

14 Cinti S. The adipose organ. Prostaglandins Leukot Essent Fatty Acids 2005; 73: 9-15

15 Palou A, Pico C, Bonet ML, Oliver P. The uncoupling protein, thermogenin. Int J Biochem Cell Biol 1998; 30: 7-11.

16 Cannon B, Nedergaard J. Brown adipose tissue: function and physiological significance. Physiol Rev 2004; 84:277-359.

17 Nedergaard J, Bengtsson T, Cannon B. Unexpected evidence for active brown adipose tissue in adult humans. Am J Physiol Endocrinol Metab 2007; 293: E444-E452.

18 Silva JE. Thermogenic mechanisms and their hormonal regulation. Physiol Rev 2006; 86: 435-464.

19 Martinez de Mena R, Scanlan TS, Obregon MJ. The T3 receptor beta1 isoform regulates UCP1 and D2 deiodinase in rat brown adipocytes. Endocrinology 2010; 151: 5074-5083.

20 Bradford MM. A rapid and sensitive method for the quantitation of microgram quantities of protein utilizing the principle of protein-dye binding. Anal Biochem 1976; 72: 248-254.

21 Pfaffl MW. A new mathematical model for relative quantification in real-time RT-PCR. Nucleic Acids Res 2001; 29: e45.
22 Nedergaard J, Cannon B. The changed metabolic world with human brown adipose tissue: therapeutic visions. Cell Metab 2010; 11: 268-272.

23 Nedergaard J, Herron D, Jacobsson A, Rehnmark S, Cannon B. Norepinephrine as a morphogen?: its unique interaction with brown adipose tissue. Int I Dev Biol 1995; 39: 827-837.

24 Giordano A, Morroni M, Santone G, Marchesi GF, Cinti S. Tyrosine hydroxylase, neuropeptide $Y$, substance $P$, calcitonin gene-related peptide and vasoactive intestinal peptide in nerves of rat periovarian adipose tissue: an immunohistochemical and ultrastructural investigation. J Neurocytol 1996; 25: 125-136.

25 Giordano A, Frontini A, Murano I, Tonello C, Marino MA, Carruba MO et al. Regional-dependent increase of sympathetic innervation in rat white adipose tissue during prolonged fasting. J Histochem Cytochem 2005; 53: 679-687.

26 Palou M, Konieczna J, Torrens JM, Sánchez J, Priego T, Fernandes ML et al. Impaired insulin and leptin sensitivity in the offspring of moderate caloricrestricted dams during gestation is early programmed. J Nutr Biochem 2012; 23: 627-639.

27 Mitchell JR, Jacobsson A, Kirchgessner TG, Schotz MC, Cannon B, Nedergaard J. Regulation of expression of the lipoprotein lipase gene in brown adipose tissue. Am J Physiol 1992; 263(3 Pt 1): E500-E506.

28 Bartelt A, Bruns OT, Reimer R, Hohenberg H, Ittrich $\mathrm{H}$, Peldschus $\mathrm{K}$ et al. Brown adipose tissue activity controls triglyceride clearance. Nat Med 2011; 17: 200-205.

29 Kerner J, Hoppel C. Fatty acid import into mitochondria. Biochim Biophys Acta 2000; 1486: 1-17.

30 Lowell BB, Spiegelman BM. Towards a molecular understanding of adaptive thermogenesis. Nature 2000; 404: 652-660.

31 Williams GR, Bassett JH. Deiodinases: the balance of thyroid hormone: local control of thyroid hormone action: role of type 2 deiodinase. J Endocrinol 2011; 209: 261-272.

32 Ribeiro MO, Carvalho SD, Schultz JJ, Chiellini G, Scanlan TS, Bianco AC et al. Thyroid hormone--sympathetic interaction and adaptive thermogenesis are thyroid hormone receptor isoform--specific. J Clin Invest 2001; 108:97-105.

33 Ribeiro MO, Lebrun FL, Christoffolete MA, Branco M, Crescenzi A, Carvalho SD et al. Evidence of UCP1-independent regulation of norepinephrine-induced thermogenesis in brown fat. Am J Physiol Endocrinol Metab 2000; 279: E314-E322.

34 de Jesus LA, Carvalho SD, Ribeiro MO, Schneider M, Kim SW, Harney JW et al. The type 2 iodothyronine deiodinase is essential for adaptive thermogenesis in brown adipose tissue. J Clin Invest 2001; 108: 1379-1385.

35 Hall JA, Ribich S, Christoffolete MA, Simovic G, Correa-Medina M, Patti ME et al. Absence of thyroid hormone activation during development underlies a permanent defect in adaptive thermogenesis. Endocrinology 2010; 151: 4573-4582.

36 Longhi S, Radetti G. Thyroid function and obesity. J Clin Res Pediatr Endocrinol 2013; 5(Suppl 1): 40-44.

37 Florez-Duquet M, McDonald RB. Cold-induced thermoregulation and biological aging. Physiol Rev 1998; 78: 339-358. 